\title{
High-throughput site-directed mutagenesis using oligonucleotides synthesized on DNA chips
}

\author{
Didier Saboulard ${ }^{1}, V_{\text {Vincent Dugas }}^{2}$, Mehdi Jaber ${ }^{2}$, Jérôme Broutin² ${ }^{2}$ Eliane \\ Souteyrand ${ }^{2}$, Julien Sylvestre ${ }^{1}$, and Marc Delcourt ${ }^{1}$ \\ ${ }^{1}$ Biométhodes SA, Evry and ${ }^{2}$ RosaTech SA, Ecully, France
}

BioTechniques 39:363-368 (September 2005)

Site-directed mutagenesis has greatly helped researchers both to understand the precise role of specific residues in coding sequences and to generate variants of proteins that have acquired new characteristics. Today's demands for more complete functional cartographies of proteins and advances in selection and screening technologies require that site-directed mutagenesis be adapted for high-throughput applications. We describe here the first generation of a library of single and multiple site-directed mutants using a mixture of oligonucleotides synthesized on DNA chips. We have used the human interleukin 15 (IL15) gene as a model, of which 37 codons were simultaneously targeted for substitution by any of eight possible codons. Ninety-six clones were sequenced, exhibiting a broad spectrum of targeted substitutions over the whole gene length with no unwanted mutations. Libraries produced using such pools of oligonucleotides open new perspectives to direct the evolution of proteins in vitro, by enabling the simple, rapid, and cost-effective generation of large tailor-made genetic diversities from any gene.

\section{INTRODUCTION}

Since the early 1980 s, our understanding of protein function has been enhanced significantly due to the advent of both site-directed mutagenesis (1) and random mutagenesis (2). Both techniques have in their own rights successfully provided useful approaches to studying protein function and engineering new proteins. Site-directed mutagenesis permits the rational assessment of the importance of individual residues and the modification of proteins in a targeted way. In a complementary manner, random mutagenesis facilitates the creation of large genetic libraries in a "blind" fashion, thus enabling a diverse set of proteins with novel or improved functions to be generated, hence giving rise to the field of directed evolution. A newer technology, Massive Mutagenesis $^{\circledR}(3)$, now combines the properties of both site-directed and random mutagenesis technologies to allow for the generation of large mutant libraries containing single or multiple site-directed mutants. Massive Mutagenesis is based on the usage of a mixture of a large number of similarly orientated mutagenic oligonucleotides, typically hundreds to thousands, in order to initiate the circular polymerization of one strand of plasmid DNA. Libraries of up to $10^{9}$ different mutants can be generated. The positions to target as well as the nature of the diversity introduced at each targeted position can be arbitrarily defined by choosing an appropriate set of oligonucleotides. Yet the technology remains limited by the cost of these oligonucleotides. If nondegenerate oligonucleotides are used, the cost per substitution is of approximately $5 €$ (equivalent to approximately $\$ 6$ U.S. dollars), which becomes an important limitation when hundreds or thousands of substitutions are sought. This cost limitation is particularly drastic when the introduction of the 19 substitutions at one position, an activity now referred to as "saturation mutagenesis" (4), is sought. We present here a straightforward and flexible way to address this problem, based on the parallel synthesis of a large number of different oligonucleotides on DNA chips and their subsequent use for Massive Mutagenesis.

\section{MATERIALS AND METHODS}

\section{Porous Silicon Formation}

A porous silicon layer was obtained by galvanostatic photoetching of polished crystalline n-type $\mathrm{Si}$ wafers [phosphorus doped; $10^{15}$ atoms $/ \mathrm{cm}^{3}$; (100) orientation] with ohmic contact on back side (Tronics, Crolles, France). The etching solution was a hydrofluoric acid (HF):ethanol (1:1, v/v) solution prepared from $49 \%$ HF in water. Samples were etched at an anodic current density of $20 \mathrm{~mA} / \mathrm{cm}^{2}$ for 6 min under $60 \mathrm{~W}$ tungsten light illumination. Under these conditions, the porous layer depth was about 10 $\mu \mathrm{m}$, and the pore diameter was approximately $0.5 \mu \mathrm{m}$. An annealing treatment at $500^{\circ} \mathrm{C}$ under dried air for $2 \mathrm{~h}$ allowed for the oxidation of the hydride terminated silicon surface and the stabilization of the porous layer with respect to subsequent harsh chemical treatments.

\section{Functionalization of Oxidized Porous Silicon Surface}

The wafers were silanized with a monofunctional silane [t-butyl 11-(dimethylaminodimethylsilyl) undecanoate] using an impregnation process (5). Subsequently, the terminal t-butyl ester functional groups were converted into the corresponding acid. The cleavage of the ester was carried out under mild conditions with iodotrimethylsilane. Samples were immersed into a $0.1 \mathrm{M}$ solution of iodotrimethylsilane in $\mathrm{CCl}_{4}$ for $12 \mathrm{~h}$ at room temperature in a dry atmosphere. This method led to an efficient capping of the silica surface because the iodotrimethylsilane reacts with residual silanol groups. Four 5'-O-DMT, N-protected nucleoside supports corresponding to the four bases (A,T,G, and C) were prepared by coupling the free $3^{\prime}$-hydroxyl protected nucleoside with the acid groups on the surface as described (6). The grafting step of the first base was controlled by infrared spectroscopy and by measuring the amount of trityl by a colorimetric technique (at $498 \mathrm{~nm}$ ). 


\section{Synthesis of Oligonucleotide Arrays on Porous Silicon Supports}

The oligonucleotides were directly synthesized on the solid supports by standard phosphoramidite chemistry. A microprojection device deposited phosphoramidite monomers and an activator at precise locations. The device consisted of a chamber that houses the DNA chip, a microprojection system coupled to an XY stage, which enabled the deposition of the phosphoramidites and activator solutions, and a reactor, which was used to perform the washing, capping, oxidation, and detritylation steps required for synthesis. The diameter of the synthesized spot was large enough to create approximately $0.1 \mathrm{pmol}$ of each oligonucleotide. Arrays of 160 oligonucleotides were manufactured. The synthesis cycle consisted of the following four repetitive steps for each layer: (i) the coupling of deoxyribonucleotide phosphoramidite to the $5^{\prime}$ end of oligonucleotide attached to the solid support. The type of nucleoside

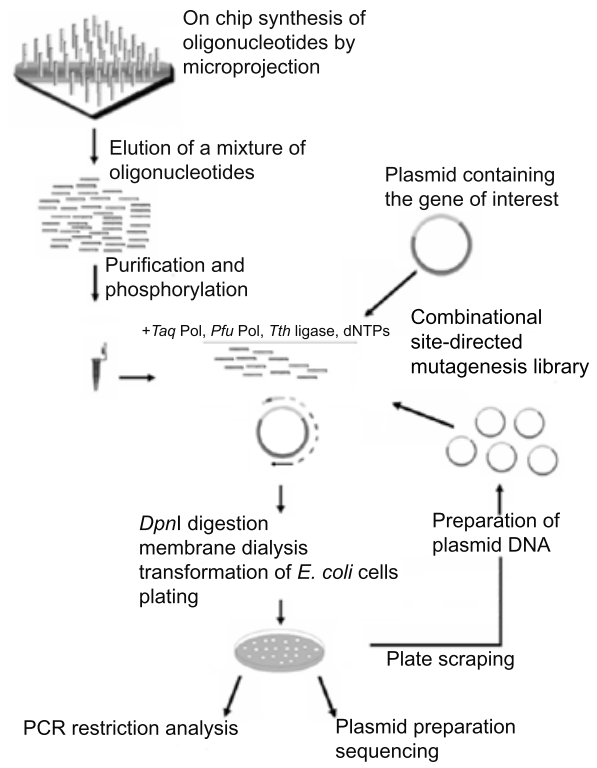

Figure 1. Representation of the protocol of Massive Mutagenesis using oligonucleotides synthesized on DNA chips. Oligonucleotides are synthesized in situ on DNA chips by microprojection. They are eluted, purified, phosphorylated, and subsequently used to introduce mutations in a plasmid by circular single-strand amplification. The product is digested using $D p n \mathrm{I}$, dialyzed, and used to transform Escherichia coli cells. Several successive rounds can be performed. Analysis of the clones is made by sequencing and/or by PCR and restriction analysis. phosphoramidite (A, C, G, or T) deposited locally was dependent on the desired sequence for each spot. The phosphoramidites require activation to react with the $5^{\prime}$-hydroxyl groups of the support-bound oligonucleotide. Tetrazole droplets (activator) were printed after the phosphoramidites droplet to perform the coupling. (ii) The capping step was used to minimize the development of truncated sequences during oligonucleotide synthesis. If some sequences did not react with the nucleotides deposited in the previous step, an acetylation process hindered them from reacting further. This process prevented the usage of the unwanted truncated sequences in Massive Mutagenesis (Biométhodes SA, Evry, France). (iii) The oxidation step used iodined reagents $[0.1 \mathrm{M}$ iodine/ pyridine/water/tetrahydrofuran (THF)] in order to stabilize the internucleotide phosphodiester bond by transforming unstable trivalent phosphor into stable pentavalent phosphor. (iv) The detritylation step "activates" the nucleotide for the next coupling step. Typically, the 5'-O-dimethoxytrityl protection group was removed by sending the $3 \%$ trichloroacetic acid/dichloromethane deblocking solution throughout the surface.

Rigorous washes with the appropriate solvent were made between each step. Steps one through four were repeated 35 times in order to synthesize the 35-mer oligonucleotides.

\section{Cleavage and Deprotection}

The synthesized oligonucleotides were removed from the surface by treatment with AMA reagent $(28 \%$ aqueous ammonium hydroxide: $41 \%$ aqueous methylamine; $1: 1, \mathrm{v} / \mathrm{v}$ ) for $4 \mathrm{~h}$ at $25^{\circ} \mathrm{C}$. Aqueous solutions were evaporated, and the resulting dry residue was suspended into ultrapure water.

\section{Oligonucleotide Purification and Phosphorylation}

Two microliters of the oligonucleotide pool were diluted in deionized water to a final volume of $100 \mu \mathrm{L}$. These oligonucleotides were then loaded on a Centricon YM3 column (Millipore, Billerica, MA, USA) and centrifuged for $40 \mathrm{~min}$ at $8500 \times \mathrm{g}$ on a tabletop centrifuge. In order to liberate $15 \mu \mathrm{L}$ of purified oligonucleotides, the column was then inverted and centrifuged for $1 \mathrm{~min}$ at $8500 \times \mathrm{g}$. Two microliters polynucleotide kinase (PNK) buffer (New England Biolabs, Beverly, MA, USA), $2 \mu \mathrm{L} 10 \mathrm{mM}$ ATP, and $1 \mu \mathrm{L} 10 \mathrm{U} / \mu \mathrm{L}$ T4 polynucleotide kinase (New England Biolabs) were added to the $15 \mu \mathrm{L}$ purified oligonucleotides. The resulting mixture was incubated for $1 \mathrm{~h}$ at $37^{\circ} \mathrm{C}$.

\section{Amplification Reaction}

The following mixture was prepared: $1 \mu \mathrm{L}$ pORF-IL15 plasmid (200 ng; Cayla-Invivogen, Toulouse, France), $1 \mu \mathrm{L} 25 \mathrm{mM}$ dNTP, $1 \mu \mathrm{L} 100$ $\mathrm{mM} \mathrm{MgSO}_{4}, 1 \mu \mathrm{L} 10 \mathrm{mM}$ ATP, $0.2 \mu \mathrm{L}$ $100 \mathrm{mM}$ NAD, $0.2 \mu \mathrm{L} 1 \mathrm{M}$ dithiothreitol (DTT), $3.5 \mu \mathrm{L} 10 \times P f u$ Pol buffer (Stratagene, La Jolla, CA, USA), 0.8 $\mu \mathrm{L} P f u$ DNA polymerase $(10 \mathrm{U} / \mu \mathrm{L}$; Stratagene), $0.8 \mu \mathrm{L}$ Tth ligase (10 $\mathrm{U} / \mu \mathrm{L} ;$ Stratagene), and $0.5 \mu \mathrm{L} T a q$ DNA polymerase $(1 \mathrm{U} / \mu \mathrm{L}$ Promega, Madison, WI, USA).

Twenty microliters phosphorylated oligonucleotides and $5 \mu \mathrm{L}$ deionized water were added to this mixture. The resulting mixture was subjected to 12 temperature cycles (at $94^{\circ} \mathrm{C}$ for $1 \mathrm{~min}$, $40^{\circ} \mathrm{C}$ for $2 \mathrm{~min}$, and $68^{\circ} \mathrm{C}$ for $20 \mathrm{~min}$ ). A complete description of the amplification protocol and possible variations is available in Reference 3.

\section{DpnI Digestion}

Four microliters $10 \times$ Buffer 4 (New England Biolabs), $0.5 \mu \mathrm{L} D p n \mathrm{I}$ (20 U/ $\mu \mathrm{L}$; New England Biolabs), and $0.5 \mu \mathrm{L}$ deionized water were added to the 35 $\mu \mathrm{L}$ of the previously amplified products and incubated for $30 \mathrm{~min}$ at $37^{\circ} \mathrm{C}$.

\section{Transformation}

Eight microliters $D p n I$ digest were dialyzed against deionized water on an MF-Millipore ${ }^{\mathrm{TM}}$ hydrophilic membrane filter $(0.025 \mu \mathrm{m}$ pore; Millipore) for 30 min. Dialyzed DNA was electroporated into $40 \mu \mathrm{L}$ electrocompetent DH10B cells and plated on LB Amp media. After overnight growth, the bacterial layer was harvested using $2 \mathrm{~mL} \mathrm{LB}$, 
and its resulting DNA was prepared using QIAquick ${ }^{\circledR}$ Miniprep kit (Qiagen, Valencia, CA, USA). Two additional rounds of mutagenesis using the same protocol and the same oligonucleotide mixture were performed.

\section{Plasmid Preparation and Sequencing}

Plasmids were prepared using the QIAquick Miniprep kit. The plasmids were sequenced using an ABI PRISM ${ }^{\circledR}$ 3100 DNA sequencer (Applied Biosystems, Foster City, CA, USA). The sequencing primer was IL15+ $\left(5^{\prime}\right.$ CTTGGAGCCTACCTAGACTCA-3').

\section{RESULTS AND DISCUSSION}

An expression vector containing the human interleukin 15 (IL15) gene was chosen as a model system to investigate the use of oligonucleotides synthesized on DNA chips for high-throughput site- directed mutagenesis. Substitutions were targeted at 37 different positions of the IL15 coding sequence. We designed eight different oligonucleotides to introduce eight substitutions (GCG, TTC, ATT, CTG, CCG, GTG, TGG, or ATG) at each of the 37 positions. The list of the 296 oligonucleotides used is presented in Supplementary Figure $\mathrm{S} 1$ available at www.BioTechniques. com. The oligonucleotides were simultaneously synthesized via classical phosphoramidite chemistry (7-9) on a solid support using a proprietary in situ DNA chip synthesizer (described in detail in the Materials and Methods section), which is based on a very flexible microprojection method (10,11).

Two major modifications were made to the in situ synthesis process in order to cost-effectively produce oligonucleotides suited for mutagenesis. First, a porous silicon substrate rather than a microscope glass slide was used to achieve a 10 - to 100 -fold increase in the quantity of oligonucleotide synthesized in a defined spot area. Second, a base-cleavable linker that permitted the elution of oligonucleotides from the surface of the porous silicon was grafted to the substrate prior to the oligonucleotide synthesis. The 296 oligonucleotides (35-mers) were simultaneously synthesized on a total surface of $8 \mathrm{~cm}^{2}$ and eluted in a volume of $10 \mu \mathrm{L}$. Approximately $30 \mathrm{pmol}$ oligonucleotides with terminal $3^{\prime}$ - and $5^{\prime}$-hydroxyl groups were recovered.

The eluted oligonucleotides were then used for Massive Mutagenesis (3), which is outlined in Figure 1. Briefly, after purification and enzymatic phosphorylation, the oligonucleotide mixture is used for a linear 12-cycle amplification reaction using a mixture of thermostable enzymes ( $P f u$ and $T a q$ DNA polymerases and Tth ligase). The oligonucleotides are all homologous to the same strand of the plasmid DNA, which avoids exponential amplification that can generate biases in favor

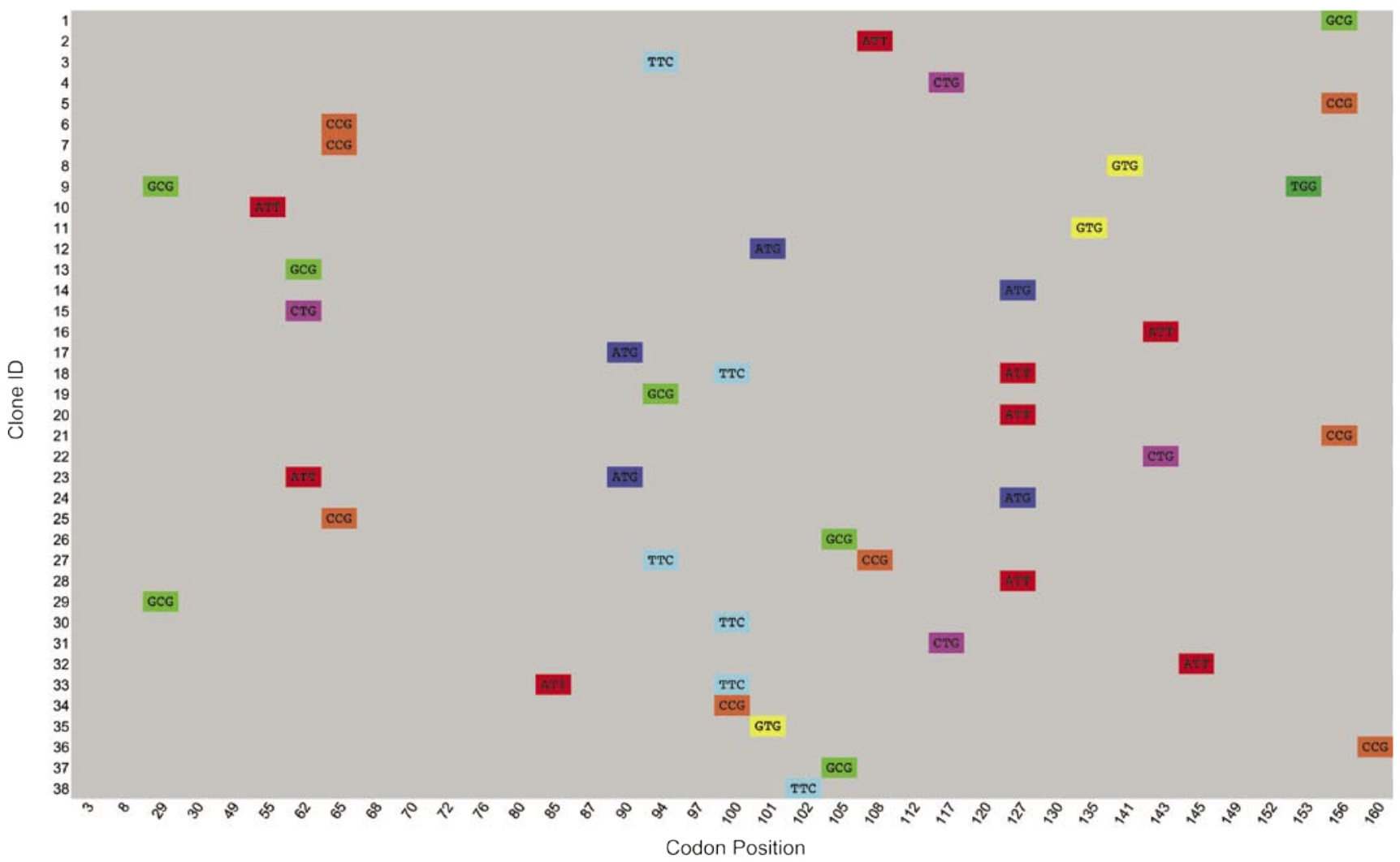

Figure 2. Sample of the diversity obtained using oligonucleotides synthesized on DNA chips and used for Massive Mutagenesis of the interleukin 15 (IL15) gene. Ninety-six clones were randomly picked and sequenced. Thirty-three clones showed one mutation. Five clones had two mutations. The various substitutions observed for these clones are represented here using an arbitrary color code. The other 58 clones exhibited no mutations. For clarity, they are not represented here. No untargeted mutation was observed among the 96 sequenced clones. 
of mutations incorporated during the first cycles. Our method is hence different from existing mutagenizing plasmid amplification protocols that use oligonucleotides homologous to both strands of the plasmid (e.g., see Reference 12) and is particularly well suited to the use of a large number of oligonucleotides. After mutagenesis, no DNA purification or double-strand ligation steps are performed, since the procedure is carried out on circular DNA. The newly synthesized DNA is treated with the methylation-sensitive restriction enzyme $D p n \mathrm{I}$, which cleaves methylated parent plasmids but does not cut the newly in vitro-synthesized hemimethylated DNA, therefore selecting for mutant products (13). The sample is then dialyzed and transformed into Escherichia coli electrocompetent cells. The following day, the bacterial colonies are harvested by scraping, and the plasmid DNA is prepared using standard procedures. In this case, a second round of mutagenesis was conducted in a similar manner using this DNA as a template, and a third round of mutagenesis was also applied. Approximately 10,000 colonies were recovered after the third round of mutagenesis.

\section{Analysis of the Mutant Library}

Ninety-six clones were randomly picked from the library and sequenced. From these 96 clones, 58 contained no mutation, 33 contained one sitedirected mutation, and 5 contained two site-directed mutations, which gives an average of 0.45 mutations per clone. One could expect the average number of mutations per molecule to be higher. Although this aspect is not fully understood, bacterial mismatch repair systems probably contribute to explain the observed mutation rate. We are indeed currently working on a modified version of Massive Mutagenesis that decreases significantly the number of nonmutant molecules obtained. Figure 2 shows the results obtained for the 38 mutant clones. The proportion of nonmutant, single-mutant, and double-mutant clones agrees well with a binomial distribution. Twenty-two out of the 37 targeted positions appear mutated in at least one clone. Each of the eight selected codon substitutions was observed, with the following number of occurrences: GCG, 7; TTC, 6; ATT, 9; CTG, 4; CCG, 8; GTG, 3; TGG, 1; and ATG, 5. Overall, the sequences presented in Figure 2 clearly show that the mutations are well spread throughout the entire length of the gene and that a broad variety of substitutions have been introduced. Although some position-specific incorporation bias seems present at a limited number of positions, the sample size did not permit us to address this issue extensively.

As an additional analysis, 192 clones were randomly selected and amplified by PCR using flanking oligonucleotides. The resulting PCR fragments were digested to assess the mutation content at five targeted positions that overlap a restriction site (data not shown). From the analysis of these 192 clones, we counted respectively 8 , 1,4 , and 10 modifications of the sites BglII (amino acid position 62), MlyI (position 101), SspI (position 143), and $B s r \mathrm{GI}$ (positions 152 or 153). These 23 clones identified by restriction analysis were confirmed by sequencing. Moreover, four of these clones were double mutants that contained one additional site-directed mutation, and three were triple mutants that contained two additional site-directed mutations, indicating that the combination of several mutations is not an exceptional occurrence in the library.

Importantly, no untargeted mutation was observed over the whole set of 96 sequenced clones. This "perfect" result on $35 \times 96=3360$ bases actually surprised us, when compared with results obtained with standard oligonucleotides. It clearly indicates that the chip-eluted oligonucleotides used in this study are of very good quality for performing mutagenesis. Additionally, the linear nature of the amplification reaction appears to have efficiently prevented the generation of polymerization-induced errors on the rest of the sequences. One could nevertheless expect a small rate of untargeted mutations to be observed, should the number of sequences sampled be larger.

\section{Conclusion}

We have reported here a very simple and straightforward method that allowed us to achieve high-throughput site-directed mutagenesis of a gene using oligonucleotides derived from chip-based synthesis. Mixtures of oligonucleotides synthesized on DNA chips have recently found an application in the domain of gene synthesis (14-16). We demonstrated that these oligonucleotide mixtures are also extremely valuable for high-throughput site-directed mutagenesis. Although an extremely low quantity per oligonucleotide $(0.1 \mathrm{pmol})$ is recovered from the DNA chips, we have shown in this study that these mixtures offer an efficient solution for the generation of libraries of combinatorial site-directed mutants. Moreover, this strategy promises substantial cost savings because the cost of standard oligonucleotide synthesis scales almost linearly with the number of oligonucleotides, whereas a chipbased approach permits the synthesis of large oligonucleotide pools at a fixed cost. It is therefore expected that, with increasing oligonucleotide number, the cost of oligonucleotides synthesized on custom DNA chips will soon become dramatically lower than the cost of those synthesized using commercial, automated solid-phase DNA synthesizers.

A detailed prediction of the cost for a typical large-scale mutagenesis project would depend on the particular in situ DNA chip synthesizer used. Although we have not tried using any other devices, we expect that any DNA chip platform that produces sufficient amounts of oligonucleotides of reasonable quality should yield equivalent results. For our own system, we estimate the cost of synthesizing one chip containing 10,000 oligonucleotides (which would allow a complete saturation mutagenesis of 526 residues) would cost approximately $1000 €$ (equivalent to approximately $\$ 1200$ U.S. dollars). In this case, the price per project would be about 50 times cheaper than conventional synthesis $(526 \times 19 \times 5 / 1000)$. This estimate does not take into account the initial system setup costs [estimated at some $25,000 €$ (or $\$ 30,000$ U.S. dollars)]. However, 
DNA chip synthesizers are commonly used for microarray production, and therefore, existing equipment may be repurposed for mutagenic oligonucleotide production.

Our approach is readily adaptable to accommodate the generation of deletions or insertions as well as that of simultaneous modifications. In the future, we would like to further explore the merits and limits of this approach, especially regarding the library sizes and the number of mutations per molecule achievable.

Applications of this technology to protein engineering should be immediate and numerous and include: (i) combinatorial saturation mutagenesis of a large number of residues of a gene in a single reaction; (ii) exhaustive amino acid scanning; and (iii) "smart" mutagenesis of proteins of known structure to maximize "interesting" diversity and to minimize the disruption of secondary structure modules

\section{ACKNOWLEDGMENTS}

We thank Stéphane Blésa for helpful discussions and Pouya Rezaeifakhr and Jean-Christophe Barale for manuscript review.

\section{COMPETING INTERESTS STATEMENT}

D.S., J.S., and M.D. are employed by Biométhodes SA, which owns the Massive Mutagenesis technology; M.D. co-invented the Massive Mutagenesis. V.D., M.J., J.B., and E.S. are employed by RosaTech SA, manufacturer of the in situ DNA chip synthesizer under license from the Centre National de la Recherche Scientifique (CNRS).

\section{REFERENCES}

1. Winter, G., A.R. Fersht, A.J. Wilkinson, M. Zoller, and M. Smith. 1982. Redesigning enzyme structure by site-directed mutagenesis: tyrosyl tRNA synthetase and ATP binding. Nature 299:756-758.

2.Leung, D.W., E. Chen, and D.V. Goeddel. 1989. A method for random mutagenesis of a defined DNA segment using a modified polymerase chain reaction. Technique 1:11-15.

3.Delcourt, M. and S. Blesa, inventors. Method for massive directed mutagenesis. U.S. patent application 20040048268.

4.Kretz, K.A., T.H. Richardson, K.A. Gray, D.E. Robertson, X. Tan, and J.M. Short. 2004. Gene site saturation mutagenesis: a comprehensive mutagenesis approach. Methods Enzymol. 388:3-11.

5.Dugas, V., G. Depret, Y. Chevalier, X. Nesme, and E. Souteyrand. 2004. Immobilization of single-stranded DNA fragments to solid surfaces and their repeatable specific hybridization: covalent binding or adsorption. Sens. Actuators B. Chem. 101:112121.

6.Pon, R.T. 1993. Protocols for oligonucleotides and analogs. Methods Mol. Biol. 20:469-472.

7. Blanchard, A.P., R.J. Kaiser, and L.E. Hood. 1996. High-density oligonucleotide arrays. Biosens. Bioelectron. 11:687.

8.Hughes, T.R., M. Mao, A.R. Jones, J. Burchard, M.J. Marton, K.W. Shannon, S.M. Lefkowitz, M. Ziman, et al. 2001. Expression profiling using microarrays fabricated by an ink-jet oligonucleotide synthesizer. Nat. Biotechnol. 19:342-347.

9. Butler, J.H., M. Cronin, K.M. Anderson, G.M. Biddison, F. Chatelain, M. Cummer, D.J. Davi, L. Fisher, et al. 2001. In situ synthesis of oligonucleotide arrays by using surface tension. J. Am. Chem. Soc. 123:88878894.

10.Lausted, C., T. Dahl, C. Warren, K. King, K. Smith, M. Johnson, R. Saleem, J. Aitchison, et al. 2004. POSaM: a fast, flexible, open-source, inkjet oligonucleotide synthesizer and microarrayer. Genome Biol. 5: R58.

11.Jezequel, J.-Y., M. Cabrera, J.-R. Martin, E. Souteyrand, M. Jaber, F. Bessueille, and J.-P. Cloarec. A method and device for fabricating a support carrying a plurality of different polynucleotide and/or peptide sequences. Patent WO 02/26373.
12.Chen, Z. and D.E. Ruffner. 1998. Amplification of closed circular DNA in vitro. Nucleic Acids Res. 26:1126-1127.

13.Li, F. and J.I. Mullins. 2002. Site-directed mutagenesis facilitated by DpnI selection on hemimethylated DNA. Methods Mol. Biol. 182:19-27.

14.Richmond, K.E., M.H. Li, M.J. Rodesch, M. Patel, A.M. Lowe, C. Kim, L.L. Chu, N. Venkataramaian, et al. 2004. Amplification and assembly of chip-eluted DNA (AACED): a method for high-throughput gene synthesis. Nucleic Acids Res. 32:5011-5018.

15.Zhou, X., S. Cai, A. Hong, Q. You, P. Yu, N. Sheng, O. Srivannavit, S. Muranjan, et al. 2004. Microfluidic PicoArray synthesis of oligodeoxynucleotides and simultaneous assembling of multiple DNA sequences. Nucleic Acids Res. 32:5409-5417.

16.Tian, J., H. Gong, N. Sheng, X. Zhou, E. Gulari, X. Gao, and G. Church. 2004. Accurate multiplex gene synthesis from programmable DNA microchips. Nature 432:1050-1054.

Received 20 April 2005; accepted 8 June 2005.

Address correspondence to Julien Sylvestre, Biométhodes SA,Genavenir 8, 5 rue Henri Desruères, F-91030 Evry, France. e-mail: julien.sylvestre@gmail.com

To purchase reprints

of this article, contact

apfeffer@BioTechniques.com 